\title{
Health-related quality of life trajectory of treatment-naive patients with Merkel cell carcinoma receiving avelumab
}

\author{
Murtuza Bharmal*,1 (iD, Sandra Nolte ${ }^{2,3}$ iD, Céleste Lebbé ${ }^{4}$, Laurent Mortier ${ }^{5}$, Andrew S \\ Brohl $^{6}$, Nicola Fazio ${ }^{7}$, Jean-Jacquez Grob ${ }^{8}$, Sara Pusceddu ${ }^{9}$, Glenn J Hanna ${ }^{10}$, Jessica C \\ Hassel $^{11}$ (D) Felix Kiecker ${ }^{3}$, Barbara Ellers-Lenz ${ }^{12}$, Marcis Bajars ${ }^{13}$, Gülseren Güzel ${ }^{12}$, Paul \\ Nghiem $^{14}$, Matthias Hunger ${ }^{2}$, Michael Schlichting ${ }^{12}$, Mickaël Henry-Szatkowski² \& Sandra P \\ $D^{\prime}$ Angelo ${ }^{15}$ \\ ${ }^{1}$ EMD Serono Research \& Development Institute, Inc., Rockland, MA 02370, USA; a business of Merck KGaA, Darmstadt, Germany \\ ${ }^{2} \mathrm{ICON}$ plc, Munich, Germany/Lyon, France \\ ${ }^{3}$ Charité - Universitätsmedizin Berlin, corporate member of Freie Universität Berlin, Humboldt-Universität zu Berlin \& Berlin \\ Institute of Health, Berlin, Germany \\ ${ }^{4}$ Université de Paris, INSERM U976 \& Dermatology \& CIC, AP-HP, Saint Louis Hospital, Paris, France \\ ${ }^{5}$ Department of Dermatology, CARADERM and University of Lille, Inserm U1189, CHU Lille, Lille Cedex, France \\ ${ }^{6}$ Moffitt Cancer Center, Tampa, FL 33612, USA \\ ${ }^{7}$ Division of Gastrointestinal Medical Oncology and Neuroendocrine Tumours, European Institute of Oncology, IEO, IRCCS, Milan, \\ Italy \\ ${ }^{8}$ Aix-Marseille University, AP-HM Hospital, Marseille, France \\ ${ }^{9}$ Department of Medical Oncology, Fondazione IRCCS Istituto Nazionale dei Tumori, Milan, Italy \\ ${ }^{10}$ Dana-Farber Cancer Institute, Boston, MA 02215, USA \\ ${ }^{11}$ Department of Dermatology, Heidelberg University Hospital, Heidelberg, Germany \\ ${ }^{12}$ Merck KGaA, Darmstadt, Germany \\ ${ }^{13}$ EMD Serono Research \& Development Institute, Inc., Billerica, MA 01821, USA; a business of Merck KGaA, Darmstadt, Germany \\ ${ }^{14}$ Division of Dermatology, University of Washington Medical Center at South Lake Union, Seattle, WA 98109, USA \\ ${ }^{15}$ Department of Medicine, Weill Cornell Medical College \& Memorial Sloan Kettering Cancer Center, New York, NY 10065, USA \\ *Author for correspondence: murtuza.bharmal@emdserono.com
}

\begin{abstract}
Aim: To evaluate changes in health-related quality of life (HRQoL) in a Phase II trial (NCT02155647) of treatment-naive patients with metastatic Merkel cell carcinoma treated with avelumab (15-month follow-up). Materials \& methods: Mixed-effect Models for Repeated Measures were applied to HRQoL data (FACT-M; EQ-5D-5L) to assess changes over time. Clinically derived progression-free survival was compared with HRQoL deterioration-free survival. Results: Overall, we saw relative stability in HRQoL among 116 included patients, with nonprogression associated with statistically and clinically meaningful better HRQoL compared with progressive disease. Deterioration-free survival rates (49-72\% at 6 months, $40-58 \%$ at 12 months) were consistently higher/better compared with progressionfree survival rates $(41 / 31 \%$ at $6 / 12$ months). Conclusion: These findings show unique longitudinal HRQoL data for treatment-naive metastatic Merkel cell carcinoma patients treated with avelumab. Clinical trial registration: NCT02155647 (ClinicalTrials.gov).
\end{abstract}

First draft submitted: 30 April 2020; Accepted for publication: 12 June 2020; Published online: 17 September 2020

Keywords: FACT-M questionnaire $\bullet$ health-related quality of life $\bullet$ Merkel cell carcinoma $\bullet$ patient reported outcome - self report

\section{Background}

Merkel cell carcinoma (MCC) is a rare, neuroendocrine cancer of the skin with poor prognosis $[1,2]$, especially once the tumor has metastasized [3]. Primary treatment of MCC consists of tumor excision followed by radiation therapy. Despite local excision and radiotherapy, however, the tumor recurs in about a third of patients, with a substantial proportion of MCC patients experiencing metastatic disease [4,5]. 
Avelumab, a fully human monoclonal antibody of the immunoglobulin G1 isotype has been shown to improve treatment options for metastatic MCC, both in the first-line setting and in chemotherapy-refractory patients $[6,7]$. As an immunotherapy, avelumab is also expected to have a more favorable safety profile than cytotoxic chemotherapy regimens. Based on results from the Phase II single-arm, open-label, multicenter, international JAVELIN Merkel 200 trial (NCT02155647), avelumab became the first treatment for metastatic MCC patients to receive approval in the USA [8], the EU [9], Japan [10] and many other countries.

The JAVELIN Merkel 200 trial consists of two parts. In the first part, chemo-refractory patients were enrolled, in other words, patients had already received and failed one or more lines of chemotherapy treatment for metastatic MCC before joining the study, while treatment-naive metastatic MCC patients were included in the second part of the trial. Results from chemo-refractory patients of the trial have already been published widely, including patient's health-related quality of life (HRQoL) data while receiving avelumab treatment [7,11-13]. In the present study, we are reporting on the treatment-naive metastatic MCC patients of the JAVELIN Merkel 200 trial [6,14]. Specifically, we report the trajectory of self-reported HRQoL scores as well as HRQoL deterioration-free survival (QFS) based on 15-month follow-up data obtained from treatment-naive metastatic MCC patients.

\section{Materials \& methods}

Study design

As described above, the JAVELIN Merkel 200 trial consists of two parts, including chemo-refractory metastatic MCC patients in part 1 and patients who were naive to systemic therapy in part 2. In both parts of the trial, patients received treatment with avelumab $10 \mathrm{mg} / \mathrm{kg}$ every 2 weeks. As part of the trial, patient-reported outcomes (PRO) questionnaires were completed at baseline, week 7 and every 6 weeks thereafter until disease progression and/or treatment discontinuation. This paper is the first to report HRQoL data obtained from metastatic MCC patients receiving avelumab as first-line MCC treatment. We focus on the 15-month follow-up dataset (cutoff date: 2 May 2019).

\section{Study population}

The intention-to-treat trial population consisted of $\mathrm{n}=116$ treatment-naive metastatic MCC patients [6,14].

\section{Patient-reported outcome assessments}

The JAVELIN Merkel 200 trial included the melanoma-specific Functional Assessment of Cancer Therapy Melanoma (FACT-M) [15] and the EQ-5D-5L [16] PRO instruments. PRO data were collected electronically at baseline, week 7 and then every 6 weeks while on treatment. For those patients who had stopped treatment, an end-of-treatment visit was assessed.

The FACT-M questionnaire is a self-administered, melanoma-specific HRQoL instrument. Although specific to melanoma, it was considered suitable for application in MCC, especially in light of the dearth of MCC-specific PRO instruments. The FACT-M includes 51 items grouped into six subscale and three summary scores. In addition, three MCC-specific FACT-M scores had been developed and validated previously [12,17], are also included in this study along with the established FACT-M subscale and summary scores, leading to a total of 12 FACT-M scores presented here. For the analysis of FACT-M data, a FACT-M PRO analysis set was defined.

The EQ-5D-5L is a self-administered, generic, preference-based HRQoL instrument developed by the EuroQol group $[16,18]$. It includes five single-item dimensions, each assessed using five levels and a vertical visual analog scale (VAS) for the patients to rate their current health state. The EQ VAS was used in this study but not the EQ-5D-5L index score, as the latter is typically used in other types of analyses such as economic modeling. EQ VAS ranges from 0 (worst imaginable health state) to 100 (best imaginable health state). For the analysis of EQ VAS data, an EQ-5D-5L PRO analysis set was defined.

Both the FACT-M and the EQ-5D-5L have been validated for use in MCC [12,17,19].

\section{Statistical analysis}

The statistical analyses of HRQoL data consisted of two parts.

First, change in patients' HRQoL over time was explored. As included MCC patients had metastatic disease, one major challenge is the occurrence of missing data due to patient dropout. As a result, Mixed-effect Models for Repeated Measures (MMRM) were used that can handle missing data without losing cases [20]. Two MMRMs were fitted to model change from baseline (CFB) across all 12 FACT-M subscale, summary and MCC-specific scores 
and the EQ VAS. Both sets of MMRM included baseline value as a fixed effect covariate and a random intercept. The random intercept implies the compound symmetry structure, assuming constant intrasubject correlations. In the first set of MMRMs, we estimated the overall effect of the treatment (avelumab) on HRQoL over the course of the study, in other words, the average CFB across assessment time points. In the second set of MMRMs, we explored potential differences in HRQoL due to disease progression across all assessment time points. To assess group differences, we added 'response status' as a binary ('nonprogression' vs 'progression') fixed effect covariate to the model. The regression coefficient obtained from this analysis then describes the average change in HRQoL scores associated with nonprogression, in other words, a positive regression coefficient indicates that 'nonprogression' is associated with better/higher HRQoL compared with 'progression' and vice versa.

Second, we examined HRQoL QFS, defined as the time up to definitive HRQoL deterioration. Definitive deterioration is a change reaching or exceeding a predefined minimal important difference (MID) at least once during the study without further improvement thereafter reaching or exceeding the MID for HRQoL improvement from baseline. QFS is reported in two ways: median QFS (in months), in other words, the time until 50\% of patients showed definitive HRQoL deterioration; QFS rates at different landmarks, in other words, the percentage of patients without definitive HRQoL deterioration at 6 and 12 months, respectively. Median QFS and QFS rates were estimated using the Kaplan-Meier method; obtained estimates were then qualitatively compared with median progression-free survival (PFS) and PFS rates, respectively, for context.

For the definition of HRQoL deterioration as applied in the statistical analyses, specific FACT-M 'minimum' and 'maximum' MID thresholds had been derived for application in MCC [12,17]. For the purpose of the present analyses, we only focus on the more conservative minimum MID. Further details on the FACT-M MID thresholds are reported elsewhere [17].

\section{Results}

Study population, sociodemographic/clinical characteristics of patients

A total of $n=116$ treatment-naive MCC patients participated in the JAVELIN Merkel 200 trial [6,14]. Of these, $\mathrm{n}=98$ patients provided valid FACT-M data at baseline, while $\mathrm{n}=100$ patients provided valid EQ-5D-5L data at baseline.

Table 1 shows the sociodemographic and clinical characteristics of patients who provided PRO data $(n=100)$. Over two thirds of MCC patients were male; average age was 73 years. The majority of patients were recruited in Western Europe (61.0\%) and 27.0\% in North America. Patients had received their MCC diagnosis of metastatic disease within about two and a half months of being included in this study. Excision of the primary MCC tumor was between 1 and 3 years earlier. Compliance rates for the FACT-M ranged from 75.6 to $87.9 \%$ between baseline and week 61 , in other words, the assessment time point closest to the 15-month data cutoff date.

\section{HRQoL over time using MMRM across all metastatic MCC patients}

As shown in Table 2, the results of the MMRM analysis of mean change from baseline over time across all assessment time points suggest relative stability in HRQoL in patients still part of the study. That is, half $(6 / 12)$ of the FACT-M subscale, summary and MCC-specific scores and the EQ VAS score show a p-value $>0.05$ for CFB, indicating overall stability of HRQoL across scales. Of the remaining six FACT-M scores showing a p-value of $\mathrm{p}<0.05$, four scores suggest some deterioration. These scores were mostly related to physical and functional subscales, with two scores reaching respective MID threshold suggesting an important deterioration over time (i.e., functional well-being, Trial Outcome Index). In contrast, two FACT-M scores (emotional well-being, psychological impact) suggest a small overall improvement, with the former subscale exceeding the MID threshold suggesting an important improvement in emotional well-being over time in those patients still part of the study.

\section{Change in HRQoL associated with 'progression' versus 'nonprogression'}

As shown in Figure 1, 'nonprogression' was associated with substantially higher/better HRQoL scores compared with 'progression' across all FACT-M subscale, summary and MCC-specific scores and the EQ VAS score. The only exception was the FACT-M melanoma surgery subscale, where the $95 \% \mathrm{CI}$ for the group difference included zero (see Figure 1; the 95\% CI starts slightly below the x-axis for this subscale). All remaining group differences were generally of a large magnitude, in other words, reaching respective MID threshold in all but two FACTM subscales (i.e., melanoma surgery subscale, physical function; see Figure 1; the horizontal MID line is at or above the respective bar of the two subscales). These findings suggest that PRO data clearly differentiated between 
Table 1. Sociodemographic/clinical characteristics of treatment-naive patients of JAVELIN Merkel 200 trial,

patient-reported outcomes analysis set population $(n=100)$.

Sociodemographic/clinical characteristics $n(\%)$

Sex

- Male $69(69.0 \%)$

- Female $\quad 31(31.0 \%)$

Age (years)

- Mean (SD) $72.83(9.66)$

- Median $\quad 74.00$

$\begin{array}{ll}\text { - Range } & 45.00-93.00\end{array}$

Pooled geographic region

- North America $\quad 27(27.0 \%)$

- Western Europe $61(61.0 \%)$

- Australasia $9(9.0 \%)$

- Asia $3(3.0 \%)$

ECOG PS at baseline

$\begin{array}{ll}-0 & 64(64.0 \%)\end{array}$

$-1 \quad 36(36.0 \%)$

Site of primary tumor

$\begin{array}{ll}- \text { Non-skin } & 5(5.0 \%)\end{array}$

- Skin $\quad 91(91.0 \%)$

- Missing $4(4.0 \%)$

Tumor size at baseline $(\mathrm{mm})$

$-\mathrm{n}$ (missing) 99 (1)

- Mean (SD) $79.98(57.49)$

- Median 66.00

$\begin{array}{lr}\text { - Range } & 0.00-288.00\end{array}$

Time since initial diagnosis (years)

$\begin{array}{ll}- \text { Mean (SD) } & 2.31(0.73)\end{array}$

- Median $\quad 2.00$

- Min-max $1.00-3.00$

Time since first metastatic disease (months)

- Mean (SD) $\quad 5.54$ (7.71)

- Median $\quad 2.45$

- Min-max $\quad 0.36-49.58$

ECOG PS: Eastern Cooperative Oncology Group performance status; SD: Standard deviation.

'progression' and 'nonprogression', with the latter showing statistically significant and clinically meaningful better HRQoL over time compared with the former in EQ VAS and almost all FACT-M scales.

\section{Median QFS}

Figure 2 presents the time until definitive deterioration based on FACT-M and EQ VAS scores, respectively. That is, we estimated the length of time (in months) until half of the patients indicated a deterioration in their HRQoL scores.

Compared with median PFS, which was 4.1 months (95\% CI: 1.4-6.1 months) for treatment-naive MCC patients [21], median QFS was longer across all HRQoL scores, with some being substantially longer than the PFS (indicated by the generally higher QFS bars in Figure 2 compared with the PFS bar). For the FACT-M scores, the shortest median QFS was observed for physical well-being, functional well-being, the melanoma subscale and the FACT-M Trial Outcome Index, respectively, with half of the patients presenting a definitive deterioration in terms of HRQoL around 4.5-5.8 months after their first treatment dose. Censoring rates ranged from $45 \%$ (physical well-being) to up to $66 \%$ (psychological impact). Due to a large amount of censoring, median QFS could not be estimated for emotional well-being and psychological impact. 


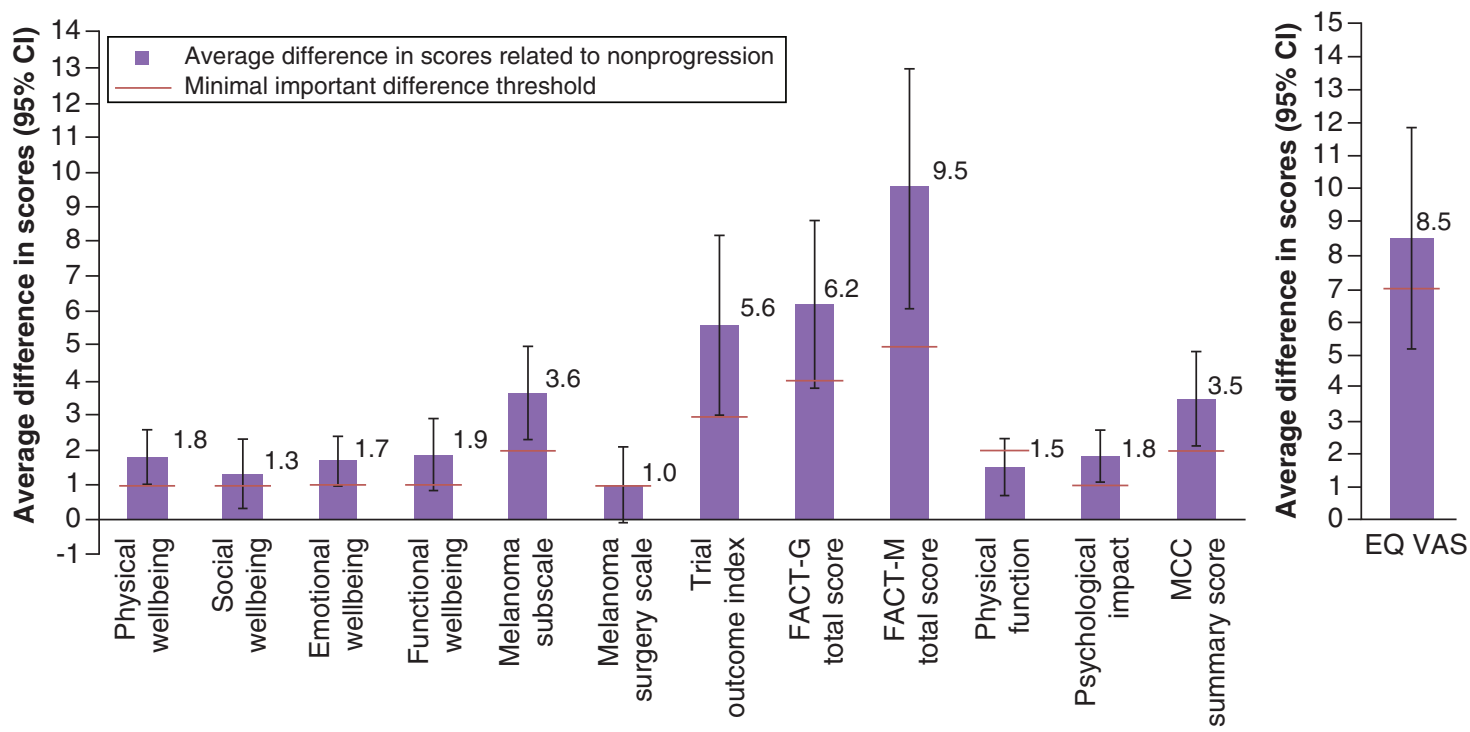

Figure 1. Average difference in scores related to nonprogression (magnitude of respective bars show by how much patients with nonprogression reported higher health-related quality of life compared with patients with progression).

FACT-M: Functional Assessment of Cancer Therapy - Melanoma; FACT-G: Functional Assessment of Cancer Therapy General; MCC: Merkel cell carcinoma; VAS: Visual analog scale.

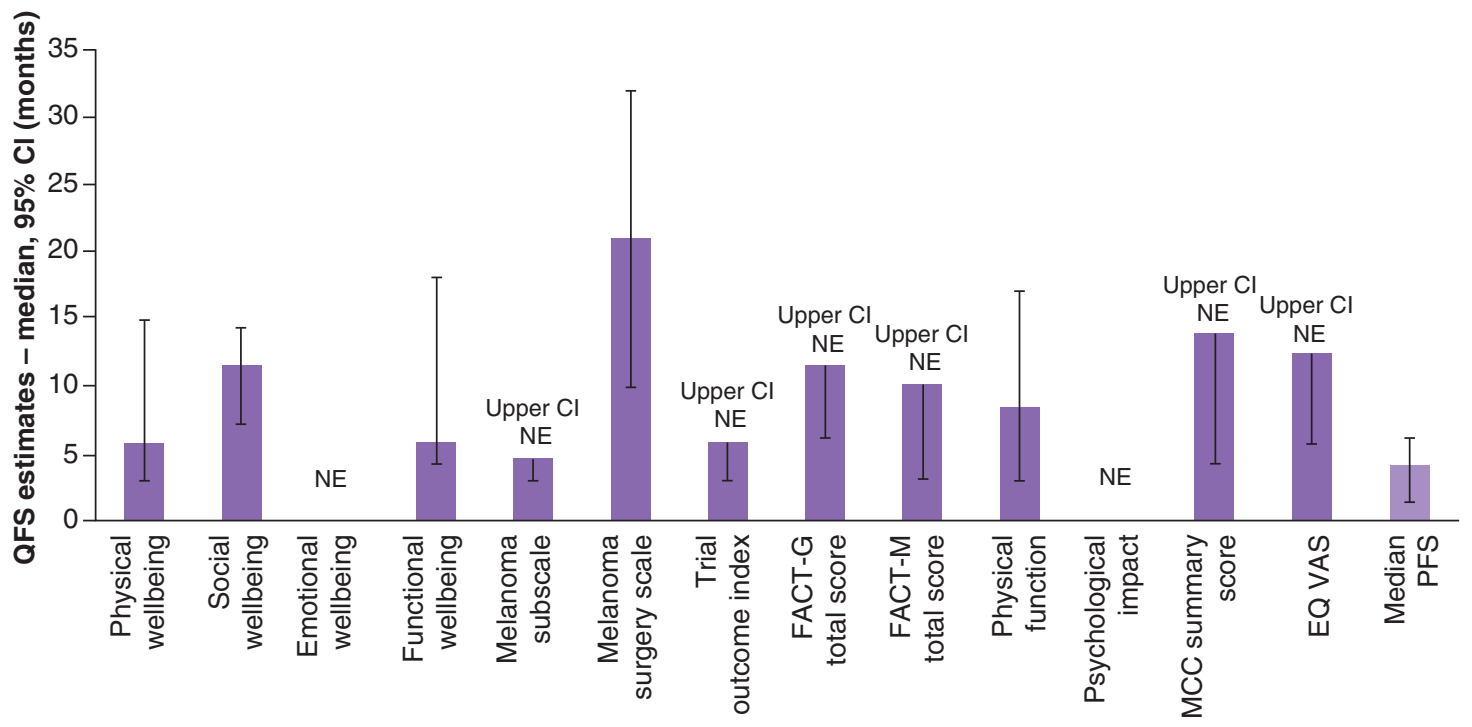

Figure 2. Health-related quality of life-based deterioration-free survival in terms of time to definitive deterioration based on Functional Assessment of Cancer Therapy - Melanoma and EQ visual analog scale versus progression-free survival (in median months).

FACT-M: Functional Assessment of Cancer Therapy - Melanoma; FACT-G: Functional Assessment of Cancer Therapy General; MCC: Merkel cell carcinoma; NE: Not estimable; PFS: Progression-free survival; QFS: Deterioration-free survival; VAS: Visual analog scale. 
Table 2. Trajectory of health-related quality of life over time (overall effect across all visits).

\begin{tabular}{|c|c|c|c|}
\hline \multirow{2}{*}{$\begin{array}{l}\text { FACT-M subscale, summary, MCC-specific } \\
\text { and EQ VAS }\end{array}$} & \multicolumn{2}{|c|}{$\mathrm{MMRM}^{\dagger}$ with 'baseline value' as fixed-effect covariate } & \multirow[t]{2}{*}{ MID } \\
\hline & Average mean change $(95 \% \mathrm{Cl})$ & p-value & \\
\hline \multicolumn{4}{|l|}{ FACT-M } \\
\hline PWB & $-0.96(-1.72 ;-0.21)$ & $0.0125^{\ddagger}$ & 1 \\
\hline SWB & $0.11(-0.47 ; 0.69)$ & 0.7187 & 1 \\
\hline EWB & $1.03(0.45 ; 1.62)$ & $0.0006^{\ddagger}$ & 1 \\
\hline FWB & $-1.12(-2.00 ;-0.25)$ & $0.0119^{\ddagger}$ & 1 \\
\hline MS & $-0.92(-2.13 ; 0.30)$ & 0.1404 & 2 \\
\hline MSS & $0.50(-0.42 ; 1.42)$ & 0.2871 & 1 \\
\hline TOI & $-3.14(-5.67 ;-0.62)$ & $0.0149 \ddagger$ & 3 \\
\hline FACT-G total score & $-0.82(-2.99 ; 1.34)$ & 0.4539 & 4 \\
\hline FACT-M total score & $-1.88(-5.12 ; 1.35)$ & 0.2539 & 5 \\
\hline \multicolumn{4}{|l|}{ MCC-specific scores } \\
\hline PF & $-1.27(-2.08 ;-0.46)$ & $0.0022^{\ddagger}$ & 2 \\
\hline $\mathrm{PI}$ & $0.97(0.37 ; 1.57)$ & $0.0015^{\ddagger}$ & 1 \\
\hline MCC summary score & $-0.34(-1.62 ; 0.94)$ & 0.6006 & 2 \\
\hline \multicolumn{4}{|l|}{ EQ-5D-5L } \\
\hline EQ VAS & $0.36(-2.64 ; 3.36)$ & 0.8138 & 7 \\
\hline \multicolumn{4}{|c|}{$\begin{array}{l}\text { †MMRMs included baseline value as a fixed-effect covariate and a random intercept to model intra-subject correlations; } \\
\text { ‡p-value }<0.05 \text {. } \\
\text { EWB: Emotional well-being; FACT-M: Functional Assessment of Cancer Therapy - Melanoma; FACT-G: Functional Assessment of Cancer Therapy - General; FWB: Functional well- } \\
\text { being; MCC: Merkel cell carcinoma; MID: Minimal important difference; MMRM: Mixed-effect Models for Repeated Measures; MS: Melanoma subscale; MSS: Melanoma surgery } \\
\text { scale; PF: Physical function; PI: Psychological impact; PWB: Physical well-being; SWB: Social/family well-being; TOI: Trial outcome index; VAS: Visual analog scale. }\end{array}$} \\
\hline
\end{tabular}

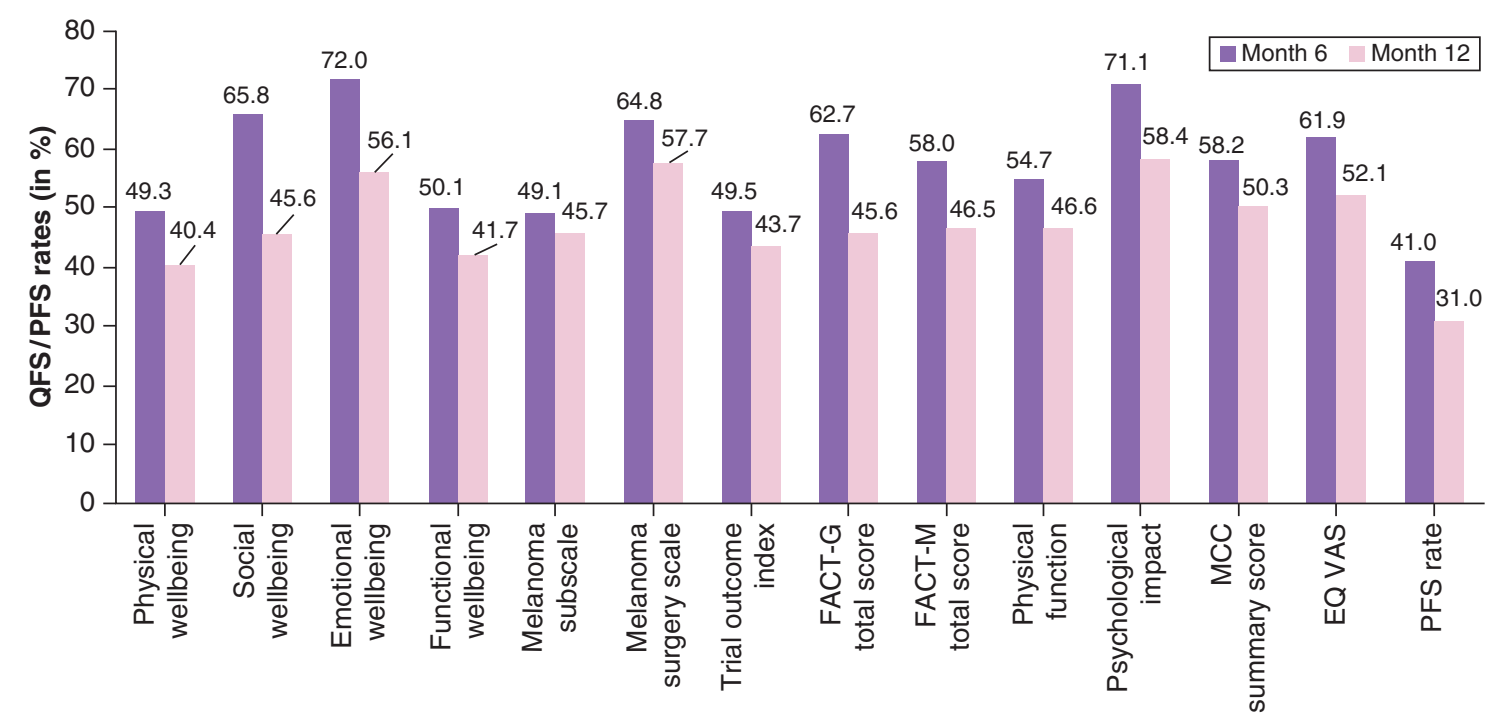

Figure 3. Health-related quality of life-based deterioration-free survival rates based on Functional Assessment of Cancer Therapy - Melanoma and EQ visual analog scale versus progression-free survival rates; 6 and 12 months. FACT-M: Functional Assessment of Cancer Therapy - Melanoma; FACT-G: Functional Assessment of Cancer Therapy General; MCC: Merkel cell carcinoma; PFS: Progression-free survival; QFS: Deterioration-free survival; VAS: Visual analog scale.

QFS rates

QFS rates show the percentage of patients without definitive HRQoL deterioration at 6 and 12 months, respectively. As shown in Figure 3, for the FACT-M subscale, summary and MCC-specific scores, QFS rates ranged from 49\% (melanoma subscale, physical well-being) to up to 72\% (emotional well-being) at 6 months. At 12 months, QFS 
rates ranged from $40 \%$ (physical well-being) to up to 58\% (psychological impact, melanoma surgery subscale). For EQ VAS, QFS rates were $62 \%$ at 6 months and $52 \%$ at 12 months, respectively. In comparison, all QFS rates were higher than PFS rates, which were $41 \%$ and $31 \%$ at 6 and 12 months, respectively [21].

\section{Discussion}

The aim of this study was to assess HRQoL of patients who took part in the JAVELIN Merkel 200 trial, a singlearm, open-label, multicenter, international Phase II study exploring the efficacy of avelumab in treatment-naive metastatic MCC patients. Specifically, we aimed to explore the trajectory of self-reported HRQoL scores based on MMRM analysis and exploring HRQoL QFS based on FACT-M and EQ VAS data. This study is the first to present PRO data obtained from treatment-naive metastatic MCC patients of the JAVELIN Merkel 200 trial.

The results of the general MMRM analysis show overall stability of HRQoL among all patients, in other words, a favorable finding, as HRQoL deterioration might be expected in patients with metastatic disease. When exploring the specific FACT-M domains, there was evidence for some improvement in emotional well-being, while there was a trend toward poorer functional and physical well-being over time. When exploring progression status, clear group differences were seen, with 'nonprogression' resulting in statistically and clinically meaningful better HRQoL compared with disease progression, in other words, differences were as expected and consistent with clinical response status.

The median duration of HRQoL QFS ranged from 4.5 months to 1 year and beyond across EQ VAS and the different FACT-M scales. Of note, the QFS of the two emotional domains emotional well-being and psychological impact was not estimable, because 'definitive deterioration' had not yet occurred for at least half of the patients at the time of data cutoff, which is in line with the results of the MMRM analyses, suggesting that patients remaining in the study seemed to be emotionally stable or even to improve over time. Compared with median PFS, median QFS was consistently longer, which was also reflected in respective QFS rates at 6 and 12 months being consistently higher than respective PFS rates at these time points.

This study is the first to present PRO data based on treatment-naive MCC patients of the JAVELIN Merkel 200 trial, while substantive quantitative and qualitative work around PRO data from chemotherapy-refractory MCC patients of the same trial has already been published extensively. Quantitative findings were largely consistent with the findings of the present study. That is, the analyses of PRO data obtained from chemo-refractory patients also showed that QFS rates were generally higher than respective PFS rates [22]. Also, the comparison of 'nonprogression' with progressive disease suggested clinically better HRQoL in nonprogressed disease in the chemo-refractory MCC cohort, like in the present study [11]. Similarly, qualitative findings based on treatment-naive MCC patients were again largely in line with the findings from chemo-refractory MCC patients in that all patients indicated similar experiences regarding perceived benefits and clinical changes during the JAVELIN Merkel 200 trial [23,24].

This study has limitations. A major challenge is the high attrition rate with substantial patient dropout throughout the trial especially within the first 6 months of study inception. While high patient dropout can be expected in metastatic disease, diminishing sample sizes result in large 95\% CIs in the QFS analyses, which are indicative of uncertainty around the estimates. A further limitation is that MMRM analyses only provide unbiased estimates if data are missing at random; however, this assumption cannot be tested empirically, so that it cannot be ruled out that the missing data pattern was nonrandom. Also, PRO data were only collected until the end of treatment, which was often triggered by disease progression with two consequences. First, an underestimation of deterioration since, for some patients, HRQoL may have deteriorated shortly after disease progression, in other words, shortly after leaving the study and second, relatively high censoring rates in the QFS analyses. Finally, we used a melanoma-specific PRO instrument in a cohort of MCC patients. MCC patients tend to be about 10 years older on average than melanoma patients, which is true for our sample with an observed mean age of 73 years. Therefore, it cannot be ruled out that especially some of the oldest participants (up to 93 years of age in our sample) found it challenging to fill out the instrument correctly. Also, some of the melanoma-specific items may not be applicable to MCC patients, as supported by our psychometric analyses where both melanoma subscales showed worse performance than the other scales [17]. However, in light of the dearth of MCC-specific PRO instruments and the exceptionally strong performance of the MCC-specific FACT-M scales physical function and psychological impact [17] gives us confidence that the use of the FACT-M in this study was appropriate and delivered high quality HRQoL data. 


\section{Conclusion}

These findings show unique longitudinal HRQoL data for treatment-naive metastatic MCC patients treated with avelumab in a relatively large sample for a rare disease. Relatively stable HRQoL scores were observed over time. When differentiating patients by progression status, 'nonprogression' was associated with statistically and clinically meaningful better HRQoL compared with progressive disease. Finally, time-to-event analyses suggest longer HRQoL QFS compared with PFS. In conclusion, these data provide important insight into self-reported HRQoL of treatment-naive metastatic MCC patients over time while receiving avelumab treatment, validating previous findings of the positive impact of avelumab treatment of patients' HRQoL among metastatic MCC.

\section{Summary points}

- This study presents unique longitudinal health-related quality of life (HRQoL) data for treatment-naive metastatic Merkel cell carcinoma (MCC) patients treated with avelumab in a relatively large sample for a rare disease.

- Compliance rates for the HRQoL data ranged from 75.6 to $87.9 \%$ between baseline and the 15 -month data cutoff date.

- In treatment-naive metastatic MCC patients treated with avelumab, nonprogression was associated with statistically and clinically meaningful better HRQoL compared with progressive disease.

- While on avelumab treatment, patients had stable HRQoL for 6 out of the 12 Functional Assessment of Cancer Therapy - Melanoma (FACT-M)/EQ visual analog scale scores considered.

- Four FACT-M scores suggested some deterioration related to physical and functional subscales, while there was evidence for some improvements in emotional well-being and the psychological impact score.

- Median HRQoL deterioration-free survival, defined as the time until a definitive deterioration in HRQoL reaching or exceeding the minimal important difference, was longer than median progression-free survival across all FACT-M/EQ visual analog scale scores considered.

- Deterioration-free survival rates at 6 and 12 months were consistently higher than respective progression-free survival rates at these time points.

- This study in treatment-naive metastatic MCC patients on treatment with avelumab validates previous HRQoL findings in patients with chemotherapy-refractory metastatic MCC.

Author contributions

SP D'Angelo, C Lebbé, L Mortier, AS Brohl, N Fazio, JJ Grob, S Pusceddu, GJ Hanna, JC Hassel, F Kiecker, B Ellers-Lenz, M Bajars, G Güzel and P Nghiem participated in data collection and review of the manuscript; $S$ Nolte reviewed and edited the analysis plan and drafted the manuscript; M Bharmal participated in the study design, analysis plan, data interpretation and drafting and review of the manuscript; $M$ Hunger reviewed and edited the analysis plan, interpreted the data and reviewed the manuscript; M Schlichting reviewed the analysis plan and the manuscript; $M$ Henry-Szatkowski conducted the analyses, interpreted the data and reviewed the manuscript.

\section{Acknowledgments}

The authors thank the patients and their families, investigators, co-investigators and the study teams at each of the participating centers and at Merck KGaA, Darmstadt, Germany and EMD Serono Research \& Development Institute, Inc., Billerica, USA; a business of Merck KGaA, Darmstadt, Germany.

\section{Financial \& competing interests disclosure}

This trial was sponsored by Merck KGaA, Darmstadt, Germany, as part of an alliance between Merck KGaA, Darmstadt, Germany and Pfizer Inc., NY, USA. M Bharmal reports employment at EMD Serono. S Nolte, M Hunger and M Henry-Szatkowski are employees of ICON plc, providing consulting services and advice to the pharmaceutical industry. C Lebbé has received honoraria from Amgen, Bristol-Myers Squibb, Incyte, Merck Sharp \& Dohme, Novartis, Pfizer, Pierre Fabre and Roche; reports serving as a consultant or advisor for Amgen, Bristol-Myers Squibb, Merck Sharpe \& Dohme, Novartis and Roche; is a member of a speakers' bureau for Amgen, Bristol-Myers Squibb, Novartis and Roche; has received research funding from Bristol-Myers Squibb and Roche; has received reimbursement for travel and accommodation expenses from Bristol-Myers Squibb; and has other relationships with Avantis Medical Systems. L Mortier has received reimbursement for travel and accommodation expenses from Bristol-Myers Squibb, Novartis and Roche/Genentech. AS Brohl reports serving as a consultant or advisor for Bayer, EMD Serono, Deciphera and PierianDx. N Fazio has received honoraria from Ipsen and Novartis; reports serving as a consultant or advisor for Advanced Accelerator Applications, Ipsen, Merck Serono, MSD Oncology, Novartis/Ipsen and Pfizer; and has received research funding from 
Merck Serono and Novartis. JJ Grob has received honoraria from Amgen, Bristol-Myers Squibb, Merck, Merck Sharp \& Dohme, Novartis, Pfizer, Pierre Fabre, Roche and Sanofi; reports serving as a consultant or advisor for Amgen, Brystol-Myers Squibb, Merck, Merck Sharp \& Dohme, Novartis, Pfizer, Pierre Fabre, Roche and Sanofi; is a member of a speakers' bureau for Novartis; and has received reimbursement for travel and accommodations expenses from Bristol-Myers Squibb, Merck Sharp \& Dohme, Novartis and Pierre Fabre. S Pusceddu has received reimbursement for travel and accommodation expenses from Novartis. GJ Hanna has received research funding from Kartos Therapeutics. JC Hassel has received honoraria from Bristol-Myers Squibb, Merck Sharp \& Dohme, Novartis, Pfizer and Roche; reports serving as a consultant or advisor for Merck Sharp \& Dohme and Pierre Fabre; has received research funding from 4SC, Amgen, BioNTech, Bristol-Myers Squibb, Immunocore, Novartis, Philogen and Roche; and has received reimbursement for travel and accommodation expenses from Pierre Fabre. F Kiecker has received honoraria from Amgen, Bristol-Myers Squibb, Merck Sharp \& Dohme, Novartis, Pierre Fabre and Roche; reports serving as a consultant or advisor for Amgen, Bristol-Myers Squibb, Incyte, Merck Sharp \& Dohme, Novartis and Roche; has received research funding from Novartis; and received reimbursement for travel and accommodation expenses from Bristol-Myers Squibb and Novartis. B Ellers-Lenz reports employment at Merck KGaA. M Bajars reports employment at EMD Serono. G Güzel reports employment at Merck KGaA. P Nghiem has received honoraria from EMD Serono and Merck Sharp and Dohme; reports serving as a consultant or advisor for EMD Serono and Pfizer; received research funding from Bristol-Myers Squibb and EMD Serono; and a pending patent for high affinity T-cell receptors that target the Merkel cell polyomavirus. M Schlichting reports employment at Merck KGaA. SP D'Angelo reports serving as a consultant or advisor for Amgen, EMD Serono, GlaxoSmithKline, Immune Design, Incyte, Merck \& Co. and Nektar; received research grants from Amgen, Bristol-Myers Squibb, Deciphera, EMD Serono, Incyte, Merck \& Co. and Nektar; and received reimbursement for travel and accommodation expenses from Adaptimmune, EMD Serono and Nektar. The authors have no other relevant affiliations or financial involvement with any organization or entity with a financial interest in or financial conflict with the subject matter or materials discussed in the manuscript apart from those disclosed.

No writing assistance was utilized in the production of this manuscript.

\section{Ethical conduct of research}

This study was performed in compliance with the ethical principles arising from the Declaration of Helsinki and all current local regulations. The study protocol was approved by Independent Ethics Committee or Institutional Review Board prior to the study launch at each site. All patients gave written informed consent.

\section{Data sharing statement}

For all new products or new indications approved in both the European Union and the USA after 1 January 2014, Merck KGaA, Darmstadt, Germany will share patient-level and study-level data after de-identification, as well as redacted study protocols and clinical study reports from clinical trials in patients. These data will be shared with qualified scientific and medical researchers, upon researcher's request, as necessary for conducting legitimate research. Such requests must be submitted in writing to the company's data sharing portal. More information can be found at https://www.merckgroup.com/en/research/our-approach-toresearch-and-development/healthcare/clinical-trials/commitment-responsible-data-sharing.html. Where Merck KGaA has a coresearch, codevelopment or comarketing/copromotion agreement or where the product has been out-licensed, it is recognized that the responsibility for disclosure may be dependent on the agreement between parties. Under these circumstances, Merck $\mathrm{KGaA}$ will endeavor to gain agreement to share data in response to requests.

\section{Open access}

This work is licensed under the Attribution-NonCommercial-NoDerivatives 4.0 Unported License. To view a copy of this license, visit http://creativecommons.org/licenses/by-nc-nd/4.0/

\section{References}

Papers of special note have been highlighted as: $\bullet$ of interest; $\bullet \bullet$ of considerable interest

1. Lemos BD, Storer BE, Iyer JG et al. Pathologic nodal evaluation improves prognostic accuracy in Merkel cell carcinoma: analysis of 5823 cases as the basis of the first consensus staging system. J. Am. Acad. Dermatol. 63(5), 751-761 (2010).

2. Schadendorf D, Lebbe C, Zur Hausen A et al. Merkel cell carcinoma: epidemiology, prognosis, therapy and unmet medical needs. Eur. J. Cancer 71, 53-69 (2017).

3. Miller NJ, Bhatia S, Parvathaneni U, Iyer JG, Nghiem P. Emerging and mechanism-based therapies for recurrent or metastatic Merkel cell carcinoma. Curr. Treat. Options Oncol. 14(2), 249-263 (2013).

4. Medina-Franco H, Urist MM, Fiveash J, Heslin MJ, Bland KI, Beenken SW. Multimodality treatment of Merkel cell carcinoma: case series and literature review of 1024 cases. Ann. Surg. Oncol. 8(3), 204-208 (2001). 
5. Soult MC, Feliberti EC, Silverberg ML, Perry RR. Merkel cell carcinoma: high recurrence rate despite aggressive treatment. J. Surg. Res. 177(1), 75-80 (2012).

6. D’Angelo SP, Russell J, Lebbe C et al. Efficacy and safety of first-line avelumab treatment in patients with stage IV metastatic Merkel cell carcinoma: a preplanned interim analysis of a clinical trial. JAMA Oncol. 4(9), e180077 (2018).

-• Reports on the effects of avelumab treatment on treatment-naive Merkel cell carcinoma (MCC) patients.

7. Kaufman HL, Russell J, Hamid O et al. Avelumab in patients with chemotherapy-refractory metastatic Merkel cell carcinoma: a multicentre, single-group, open-label, Phase II trial. Lancet Oncol. 17(10), 1374-1385 (2016).

- Reports on the effects of avelumab treatment on chemo-refractory MCC patients.

8. EMD Serono/Merck KGaA, Darmstadt, Germany and Pfizer Inc. FDA grants approval for BAVENCIO ${ }^{\circledR}$ (avelumab), the first immunotherapy approved for metastatic Merkel cell carcinoma (2020). www.pf izer.com/news/press-release/press-release-detail/fda_gra nts_approval_for_bavencio_avelumab_the_first_immunotherapy_approved_for_metastatic_Merkel_cell_carcinoma

9. Merck KGaA, Darmstadt, Germany and Pfizer Inc. European commission approves Bavencio (avelumab) for metastatic Merkel cell carcinoma (2020). www.pf izer.com/news/press-release/press-release-detail/european_commission_approves_bavencio_avelumab_for_met astatic_Merkel_cell_carcinoma

10. Merck KGaA, Darmstadt, Germany and Pfizer Inc. Bavencio (avelumab) approved for Merkel cell carcinoma in Japan (2020). www.pf iz er.com/news/press-release/press-release-detail/bavencio_avelumab_approved_for_Merkel_cell_carcinoma_in_japan

11. Kaufman HL, Hunger M, Hennessy M, Schlichting M, Bharmal M. Non-progression with avelumab treatment associated with gains in quality of life in metastatic Merkel cell carcinoma. Future Oncol. 14(3), 255-266 (2018).

-. Reports HRQoL effects of avelumab treatment on chemo-refractory MCC patients.

12. Bharmal M, Fofana F, Barbosa CD et al. Psychometric properties of the FACT-M questionnaire in patients with Merkel cell carcinoma. Health Qual. Life Outcomes 15(1), 247 (2017).

-• Provides evidence for the suitability of the PRO instruments applied in this study for use in MCC.

13. D'Angelo S, Nolte S, Schlichting M, Henry-Szatkowski M, Hennessy M, Bharmal M. Health-related quality of life in patients with metastatic Merkel cell carcinoma receiving second-line or later avelumab treatment: 36-month follow-up data. Ann. Oncol. 30(Suppl. 5), Abstract 1320P (2019).

- Reports on the effects of avelumab treatment on chemo-refractory MCC patients.

14. D'Angelo SP, Russell J, Hassel JC et al. First-line (1L) avelumab treatment in patients (pts) with metastatic Merkel cell carcinoma (mMCC): preliminary data from an ongoing study. Ann. Oncol. 30(15), 9530 (2017).

-• Reports on the effects of avelumab treatment on treatment-naive MCC patients.

15. Cormier JN, Davidson L, Xing Y, Webster K, Cella D. Measuring quality of life in patients with melanoma: development of the FACT-melanoma subscale. J. Support. Oncol. 3(2), 139-145 (2005).

16. Herdman M, Gudex C, Lloyd A et al. Development and preliminary testing of the new five-level version of EQ-5D (EQ-5D-5L). Qual. Life Res. 20(10), 1727-1736 (2011).

17. Bharmal M, Nolte S, Henry-Szatkowski M, Hennessy M, Schlichting M. Update on the psychometric properties and minimal important difference (MID) thresholds of the FACT-M questionnaire for use in treatment-naive and previously treated patients with metastatic Merkel cell carcinoma. Health Qual. Life Outcomes 18(1), 145 (2020).

-. Provides evidence for the suitability of the patient-reported outcomes (PRO) instruments applied in this study for use in MCC.

18. Euroqol G. EuroQol-a new facility for the measurement of health-related quality of life. Health Policy 16(3), 199-208 (1990).

19. Bharmal M, Hunger M, Schlichting M. Psychometric properties of EQ-5D-5L scoring algorithms for the United Kingdom in metastatic Merkel cell carcinoma. Value Health 22(10), 1170-1177 (2019).

-. Provides evidence for the suitability of the PRO instruments applied in this study for use in MCC.

20. Barnes SA, Mallinckrodt CH, Lindborg SR, Carter MK. The impact of missing data and how it is handled on the rate of false-positive results in drug development. Pharm. Stats. 7(3), 215-225 (2008).

21. D'angelo SP, Lebbe C, Mortier L et al. First-line avelumab treatment in patients with metastatic Merkel cell carcinoma: primary analysis after $\geq 15$ months of follow-up from JAVELIN Merkel 200, a registrational Phase II trial. J. Immunother. Cancer. 7(1), Abstract P362 (2019).

22. D’Angelo SP, Fofana F, Schlichting M, Henry-Szatkowski M, Hennessy M, Bharmal M. Responder analysis based on patient-reported outcomes (PROs) and clinical endpoints (CEPs) in patients (pts) with metastatic Merkel cell carcinoma (mMCC) treated with avelumab. Ann. Oncol. 29(Suppl. 8), Abstract 1282P (2018).

23. Kaufman HL, Dias Barbosa C, Guillemin I, Lambert J, Mahnke L, Bharmal M. Living with Merkel cell carcinoma (MCC): development of a conceptual model of MCC based on patient experiences. Patient 11(4), 439-449 (2018).

24. D'Angelo SP, Lambert J, Russell J et al. Patient experiences with avelumab in treatment-naive metastatic Merkel cell carcinoma: qualitative interview findings from a registrational clinical trial. J. Clin. Oncol. 37(Suppl. 8), Abstract 90 (2019). 


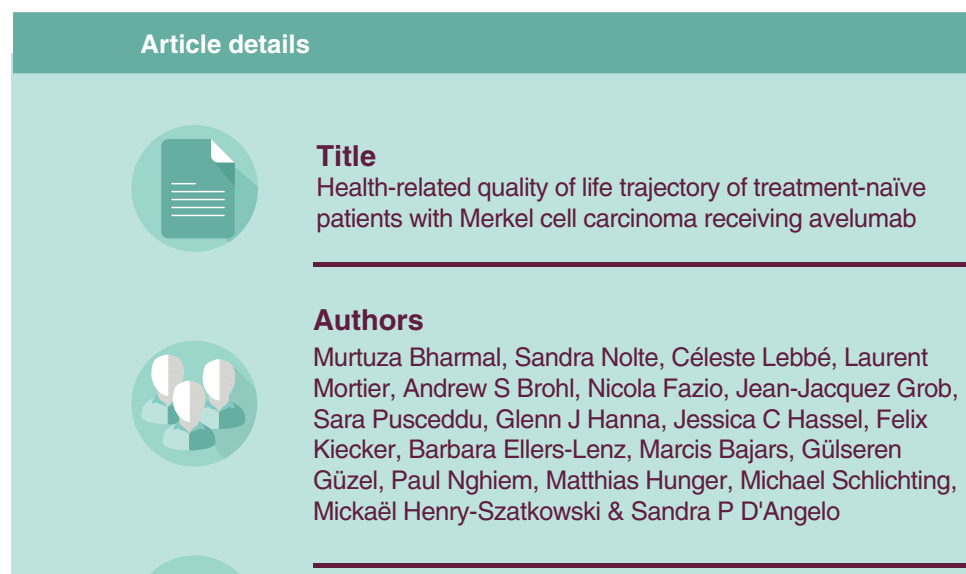

\section{Citation}

Future Oncology (2020) doi/10.2217/fon-2020-0426

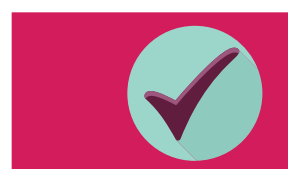

Compliance rates for the HRQoL data for those still in the study were $>75 \%$ for the entire study period

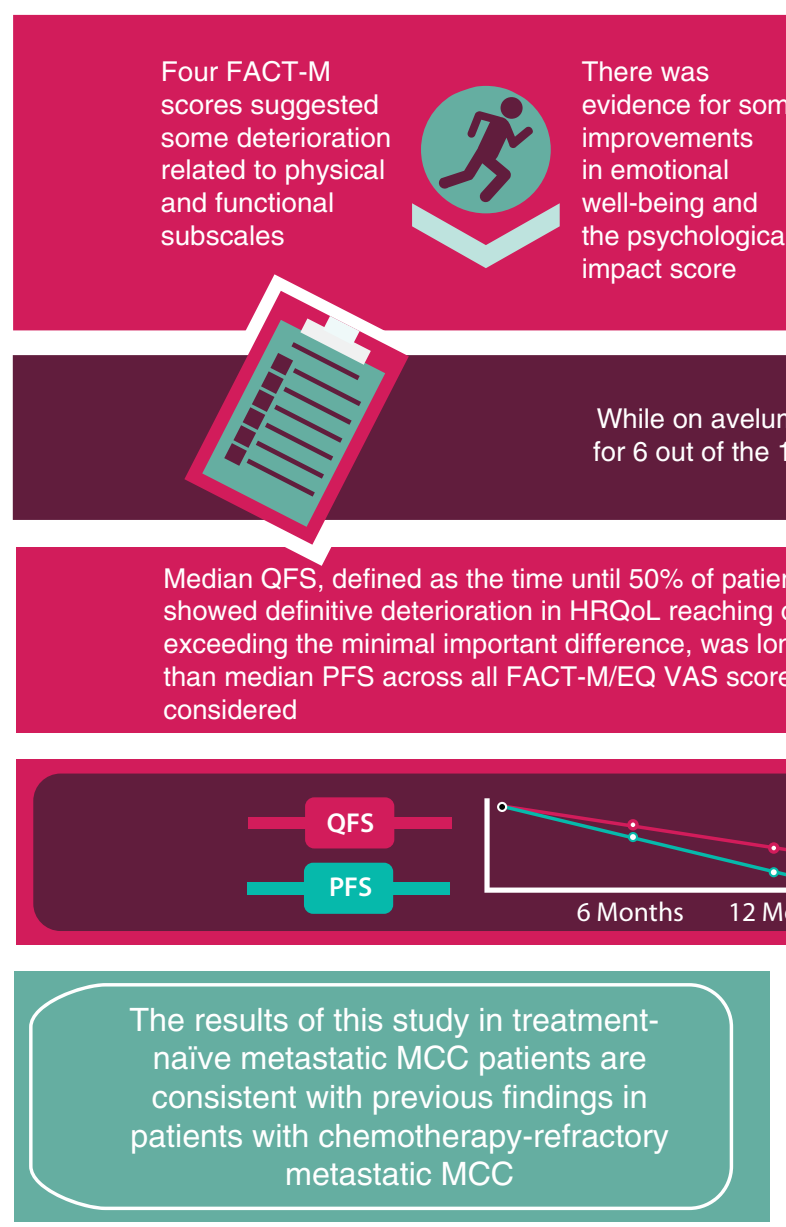

This study presents unique longitudinal HRQoL data for

treatment-naïve metastatic MCC patients treated with avelumab in a relatively large sample for a rare disease
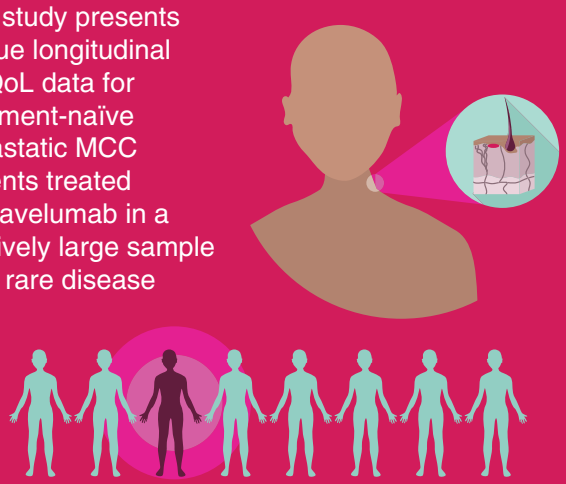

In treatment-naïve metastatic MCC patients treated with avelumab, non-progression was associated with statistically and clinically meaningful better HRQoL compared with progressive disease
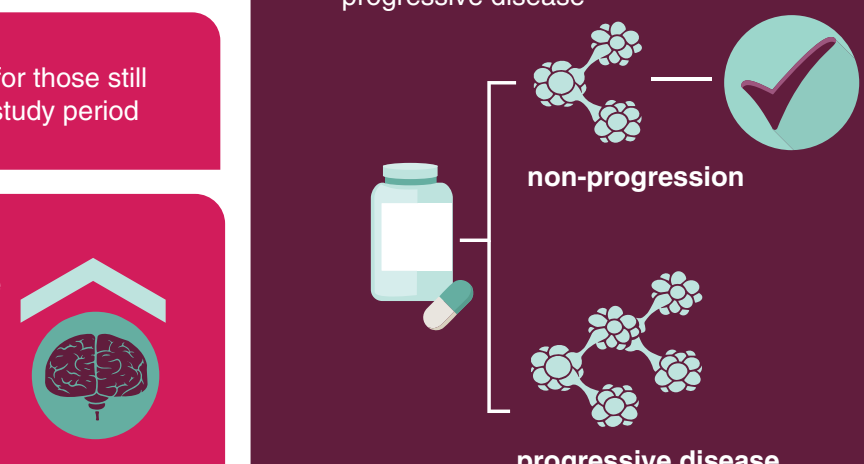

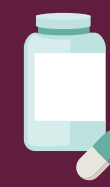

non-progression

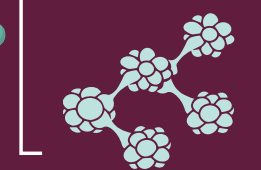

progressive disease 